Erratum

\title{
Cell cycle regulation in response to DNA damage in mammalian cells: $A$ historical perspective
}

\author{
John P. Murnane \\ Laboratory of Radiobiology and Environmental Health, Box 0750, University of California, San Francisco, \\ California, USA
}

Cancer and Metastasis Reviews 14: 17-29, 1995

An error appeared on page 21 of John P. Murnane's paper, 'Cell cycle regulation in response to DNA damage in mammalian cells: a historical perspective', which appeared in volume 14, number 1, of Cancer and Metastasis Reviews. The correct version of page 21 is found on the reverse side of this page. 